\title{
Biofilm Feedbacks Alter Hydrological Characteristic of Fractured Rock Impacting Sulfidogenesis and Treatment
}

Yiwei Cheng ${ }^{1}$, Christopher G. Hubbard ${ }^{2}$, Jil T. Geller ${ }^{1}$, Chunwei Chou ${ }^{1}$, Marco Voltolini ${ }^{1}$, Anna L Engelbrektson ${ }^{3,4}$, John D Coates ${ }^{3,4}$, Jonathan B. Ajo-Franklin ${ }^{1}$, Yuxin $\mathrm{Wu}^{1 *}$

${ }^{1}$ Earth and Environmental Sciences Area, Lawrence Berkeley National Laboratory, Berkeley, California, USA.

${ }^{2}$ Water Research Institute, Cardiff University, Cardiff, Wales.

${ }^{3}$ Energy Biosciences Institute, University of California, Berkeley, California, USA

${ }^{4}$ Department of Plant and Microbial Biology, University of California, Berkeley, California, USA

*Corresponding author: ywu3@1bl.gov

Supporting Information

6 Pages

5 Figures 
Figure S1. Thermography Calibration. Linear regression between infrared (IR) measurements (from thermal camera) and thermocouple measurements.

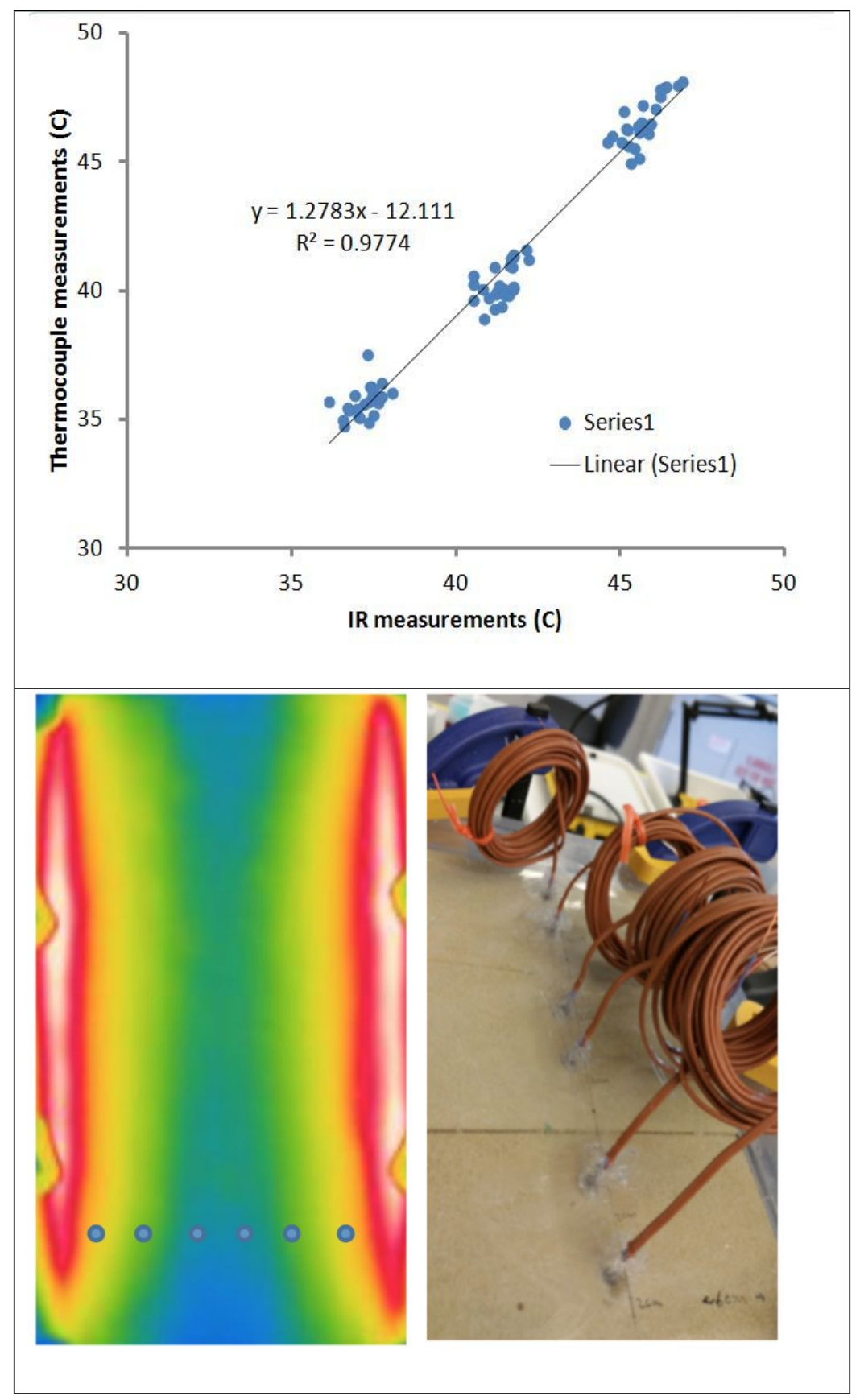


Figure S2: Results of image analysis. Time series of mean greyness value of all the pixels in the near-fracture region $\left(\mu_{\text {greyness }}\right)$.

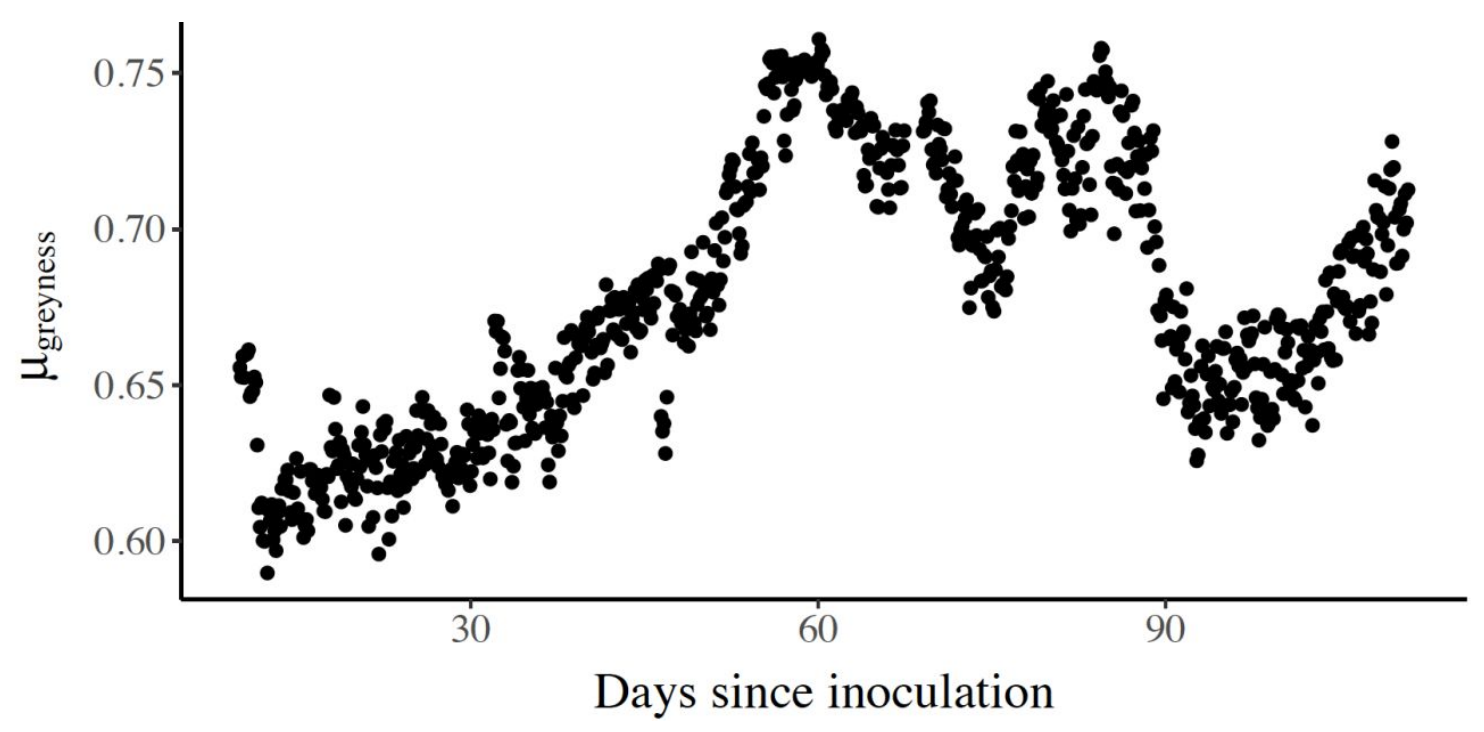


Figure S3. Normalized effluent fluorescein (red line) and salt (blue line) tracer concentrations. Flow rate is $\sim 1 \mathrm{~mL} \mathrm{~min}^{-1}$.

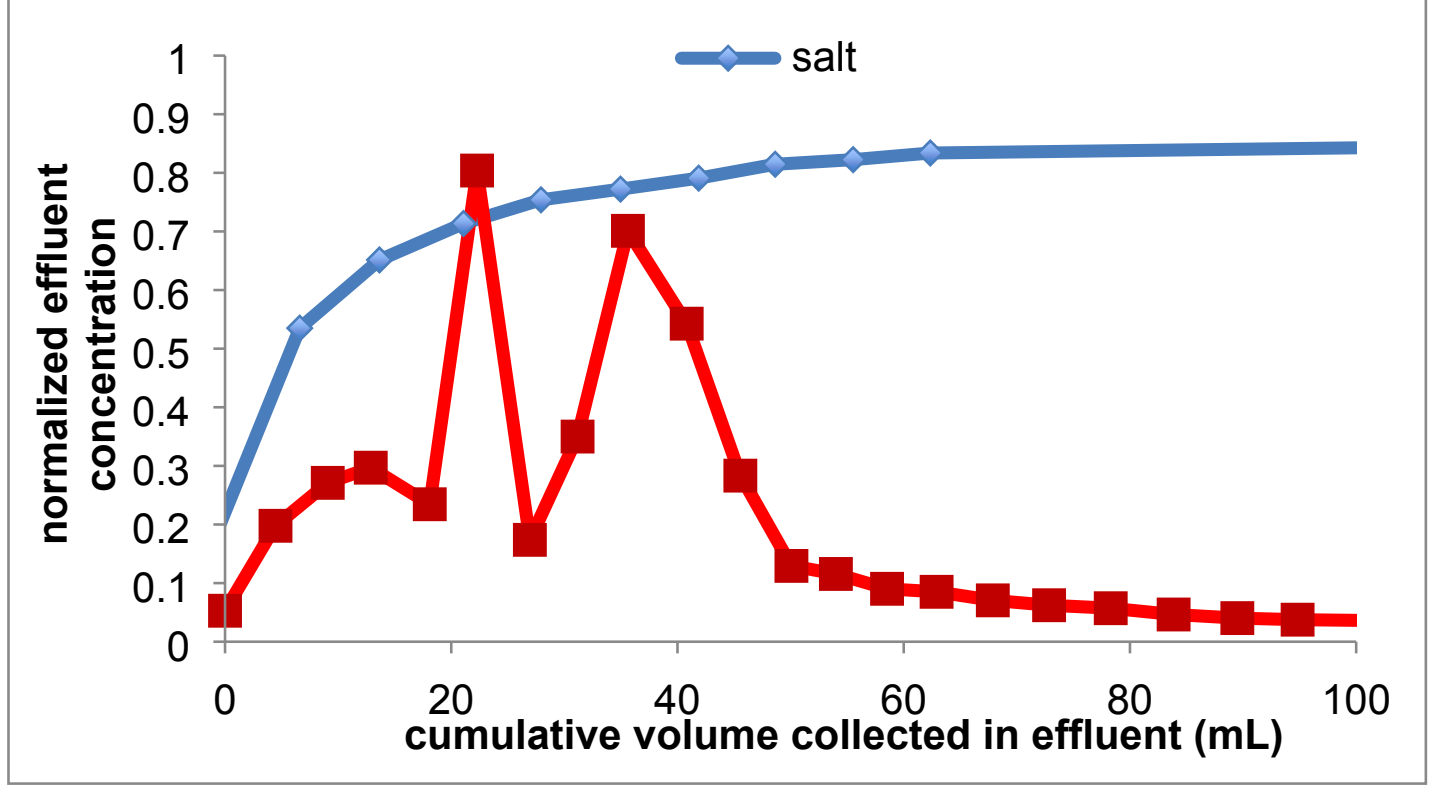


Figure S4: (A) Time series of consumption of effluent sulfate and corresponding production of effluent sulfide. (B) Time series of consumption of effluent acetate and corresponding production of effluent sulfide.
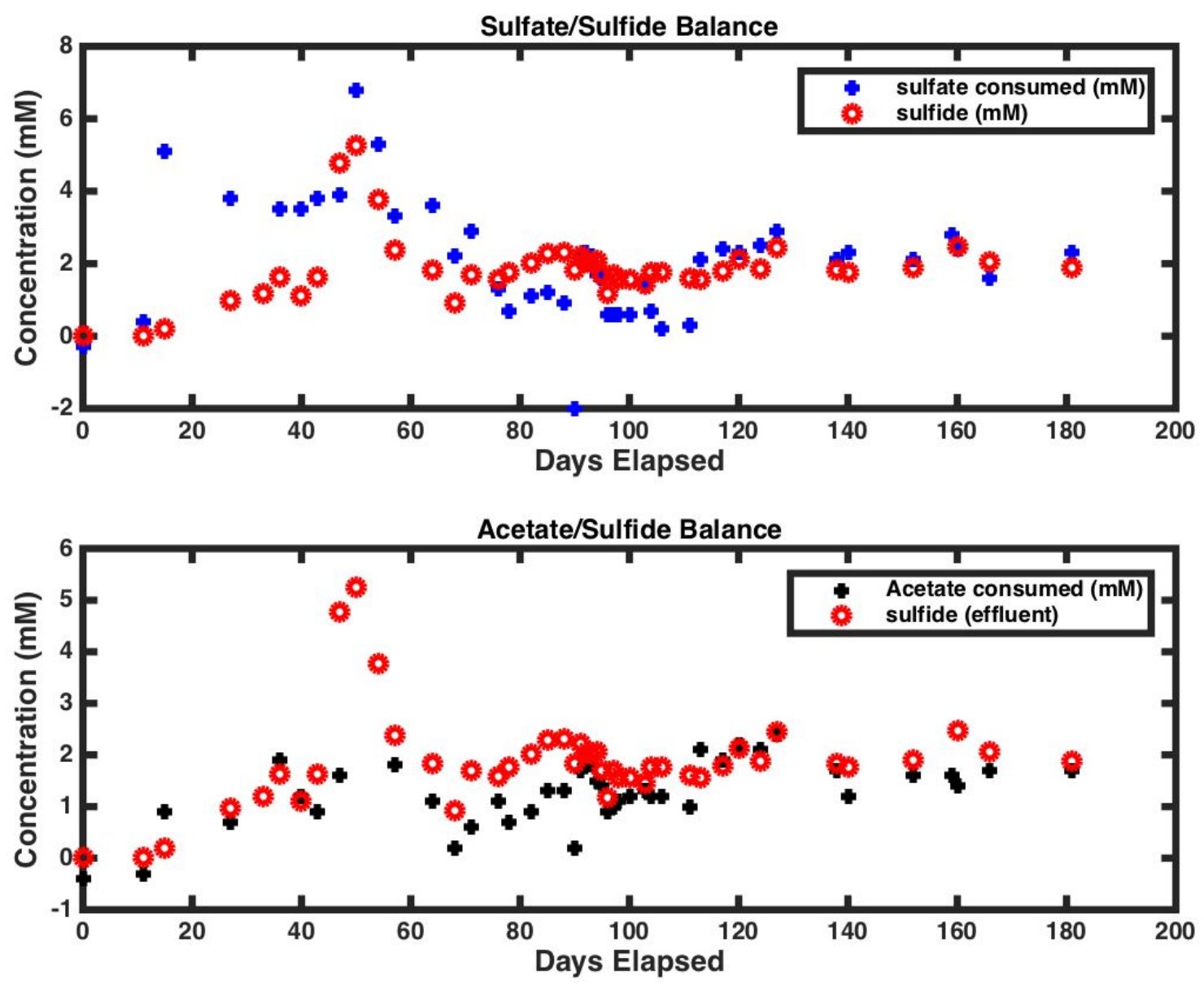
Figure S5. Dissolved sulfide, sulfate and perchlorate concentrations, of samples collected from sampling wells.
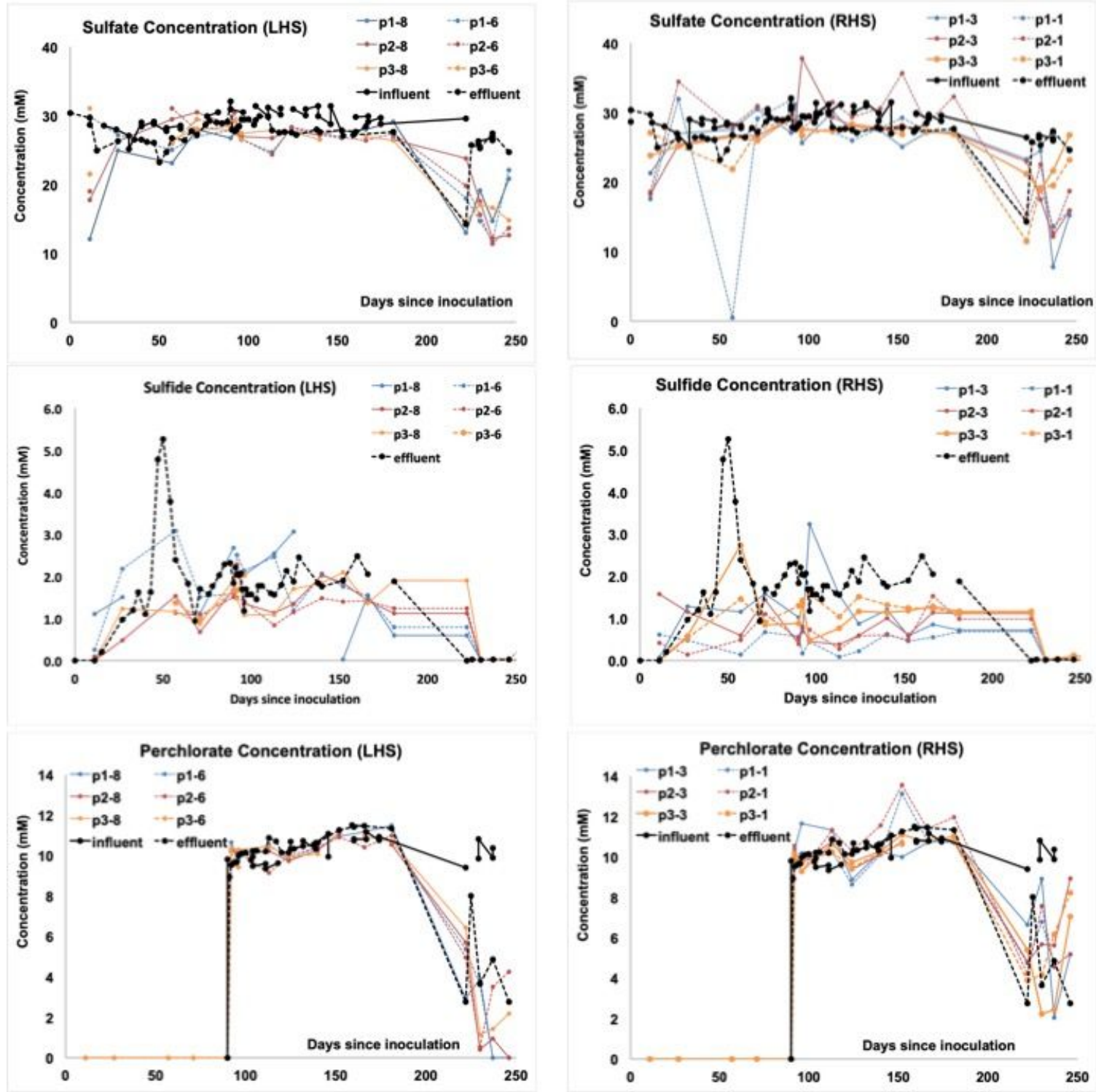